ORIGINAL

\title{
RELACIÓN DE LA CALIDAD DE VIDA CON DIFERENTES MODELOS DE ATENCIÓN DOMICILIARIA EN ENFERMOS ONCOLÓGICOS TERMINALES DE UN ÁREA SANITARIA DE MADRID (*)
}

Yolanda Agra Varela (1), Antonio Sacristán Rodea (2), Marta Pelayo Alvarez (3), Julia Fernández (4)

(1) Centro de Salud Pacífico. Area 1 IMSALUD. Madrid.

(2) Equipo de Soporte de Atención Domiciliaria. Área 4. IMSALUD. Madrid.

(3) Centro de Salud Algemesí. Area 10 SERVASA. Valencia.

(4) Equipo de Soporte de Atención Domiciliaria. Área 2. IMSALUD. Madrid.

(*) Estudio financiado por el Fondo de Investigaciones Sanitarias (FIS expte: 99/0482).

\section{RESUMEN}

Fundamentos: En este trabajo se evalúa la efectividad de los cuidados paliativos a domicilio con el objetivo de determinar si los enfermos con cáncer terminal tratados por Equipos de Soporte de Atención Domiciliaria presentan mejor calidad de vida que los tratados por Equipos de Atención Primaria.

Métodos: Estudio prospectivo cuasi experimental realizado en el Área 4 del Imsalud de Madrid con enfermos referidos desde el hospital a Equipos de Soporte de Atención Domiciliaria o a Equipos de Atención Primaria. La variable estudiada fue la calidad de vida medida a través del Rotterdam Symptom Check List y el Hospital Anxiety and Depression Scale. Para el análisis estadístico se utilizó MANOVA, ANOVA de medidas repetidas y el test de Friedman.

Resultados: 165 enfermos fueron tratados por Equipos de Soporte de Atención Domiciliaria y 56 por Equipos de Atención Primaria. Los primeros presentaron mejor puntuación en las escalas global (diferencia de medias: 9,5; IC 95\%: 2,3-16,67) y dolor (diferencia de medias: 6,4; IC 9\%: 1,14-11,43) del Rotterdam Symptom Check List en la primera semana del estudio. El tamaño del efecto fue mayor en el grupo Equipos de Soporte de Atención Domiciliaria. La prescripción de fármacos y el lugar de la muerte fue diferente en ambos grupos.

Conclusiones: Al principio del estudio los enfermos tratados por Equipos de Soporte de Atención Domiciliaria presentaron una mejor percepción del estado de salud que los tratados por Equipos de Atennúmero de pérdidas a lo largo del mismo hace que haya que mirar estos resultados con precaución.

Palabras clave: Atención primaria de salud. Calidad de vida. Atención paliativa. Cuidado terminal. Enfermo terminal. Neoplasia. Servicios de atención de salud a domicilio.

Correspondencia:

Yolanda Agra Varela

C) Reyes Magos, 21. 2807 Madrid.

Correo electrónico: yoagra@ tiscali.es
ABSTRACT

The Relationship between Quality of Life and Different Models of Home Care among Terminal Cancer Patients in an Health Area of Madrid, Spain

Background: This study assesses the effectiveness of the palliative home care for the purpose of ascertaining whether terminal cancer patients treated by Homecare Support Teams have a better quality of life than those treated by Primary Care Teams.

Methods: A quasi-experimental prospective study conducted in Madrid Healthcare District 4 on patients referred from the hospital to Homecare Support Teams or to Primary Care Teams. The main study variable was the quality of life gauged using the Rotterdam Symptom Check List and the Hospital Anxiety and Depression Scale. Statistical differences were evaluated by MANOVA, repeated measures ANOVA and Friedman test.

Results: A total of 165 patients were treated by Homecare Support Teams and 56 by Primary Care Teams. The patients treated by the Homecare Support Teams showed better point scores on the overall scale (mean differece: 9.5 ; CI 95\%; 2.3-16.67) and pain scale (mean difference: 6.4 ; CI 9\%; 1.14-11.43) on the Rotterdam Symptom Check List during the first week of the study. The effect size was greater on the Homecare Support Team group. The prescription of drugs and the place of death were different between both groups.

Conclusions: At the start of the study, the patients treated by Homecare Support Teams showed a better perception of the health condition than those treated by the Primary Care Teams. However, the great number of patients lost along the study makes it necessary be cautious when considering these results.

Keywords: Primary Health Care. Quality of life. Palliative care. Terminal care. Terminally ill. Neoplasm. Home care services. 


\section{INTRODUCCIÓN}

A pesar de los avances tecnológicos conseguidos en las ultimas décadas, los datos epidemiológicos disponibles señalan que la incidencia y mortalidad por cáncer han aumentado. Actualmente el 50\% de los enfermos diagnosticados de cáncer mueren a consecuencia del avance de su enfermedad ${ }^{1}$. Un elevado porcentaje de estos enfermos presentarán una «fase terminal» que precisará de cuidados paliativos centrados en el control de síntomas y el mantenimiento de la calidad de vida.

La tendencia actual en política sanitaria en algunos países de Europa ${ }^{2}$ es organizar la asistencia a los enfermos terminales en su domicilio y transferir los cuidados paliativos al ámbito de la atención primaria, siempre y cuando se den condiciones asistenciales básicas para ello, lo que se traduciría en mayor calidad de vida para los enfermos y menor coste para el sistema sanitario. Sin embargo la efectividad de los programas de cuidados paliativos en domicilio todavía no está bien establecida ${ }^{3,4}$.

Aunque algunos estudios han mostrado cierto beneficio en el control de síntomas por parte de unidades especificas de cuidados paliativos los datos no son concluyentes, por lo que no existe evidencia científica suficiente sobre qué tipo de asistencia a estos enfermos resulta más efectiva ${ }^{4-6}$. Por este motivo se planteó la hipótesis de que posiblemente la calidad de los cuidados paliativos sería diferente según el nivel de especialización de los profesionales que los realicen.

Este estudio se llevó a cabo con el objetivo de determinar si los enfermos oncológicos terminales incluidos en un programa de cuidados paliativos presentan mejor calidad de vida cuando son atendidos por una Unidad especializada en dichos cuidados que cuando son atendidos por los médicos de atención primaria.

\section{SUJETOS Y MÉTODO}

El diseño del estudio fue de tipo prospectivo, cuasi experimental, y se realizó en el Área 4 del Imsalud de Madrid, que atiende aproximadamente a una población de 537.000 habitantes y cuenta desde 1991 con un Programa de Cuidados Paliativos ${ }^{7}$ (PCP) para enfermos oncológicos terminales (POT). Este programa describe los criterios de inclusión, organización de las visitas y líneas generales de tratamiento y cuidados necesarios a lo largo del seguimiento de los enfermos.

La secuencia de entrada y seguimiento en PCP es la siguiente: El hospital de referencia (Hospital Ramón y Cajal) notifica al Equipo de Soporte de Atención Domiciliaria (ESAD) la existencia de un enfermo con criterios de inclusión en el programa y éste se lo comunica al medico de atención primaria responsable del mismo, quien decide si asume sus cuidados o los transfiere al ESAD. En el caso del ESAD el enfermo que entra en el programa es visitado en el plazo máximo de 48 horas por un médico y una enfermera, que establecen el tratamiento y los cuidados iniciales, así como la programación de las siguientes visitas, que suelen tener como mínimo una frecuencia semanal. Todos los profesionales del ESAD (un oncólogo, un médico general, dos enfermeras y una auxiliar de clínica) están especializados en cuidados paliativos). En el caso de EAP los enfermos son visitados a criterio del médico responsable del paciente. Los profesionales de los EAP tienen a su disposición los protocolos de actuación terapéutica que figuran en el PCP.

En este estudio se incluyó a los pacientes oncológicos terminales (POT) que entraron en programa desde el 1 de abril de 1999 hasta el 1 de marzo de 2000, afectados de enfermedad oncológica terminal (informe clínico hospitalario de enfermedad oncológica en la que existe acuerdo para no aplicar tratamientos con finalidad curativa), con esperanza de 
vida no superior a 6 meses, mayor de 18 años, con conocimiento por parte de la familia de la fase terminal de la enfermedad, que contaban con un cuidador primario (persona conviviente con el enfermo que asume sus cuidados básicos) y el consentimiento informado para participar en el estudio ${ }^{7}$. Se excluyó a los enfermos con Karnofsky Performance Status ${ }^{8}<20$, enfermos con más de tres respuestas erróneas en la escala de Pffeifer $^{9}$ y enfermos atendidos conjuntamente por ESAD y EAP.

Se eligió una muestra al azar por muestreo aleatorio simple de todos los POT que cumplieran criterios de inclusión atendidos por el ESAD durante el periodo de estudio y los atendidos por 13 EAP, elegidos al azar, de los 18 existentes en aquél momento en el Área. Los médicos de los EAP seleccionados fueron invitados a participar en el estudio a través de reuniones y cartas personales, sin conocer los objetivos del mismo. Su misión era aportar información de los enfermos que durante el periodo de estudio entraban en el programa y facilitar el acceso de los investigadores a sus datos clínicos.

La calidad de vida, principal variable de resultado de este estudio, fue medida por la versión española del Rótterdam Symptom Check List (RSCL), cuya validez, fiabilidad y sensibilidad a los cambios ha sido mostrada en estudios previos ${ }^{10,11}$. Este cuestionario consta de 39 ítems que conforman cuatro escalas (síntomas físicos, síntomas psíquicos, actividades de la vida diaria y calidad de vida global) y cuatro subescalas (fatiga, dolor, gastrointestinal y quimioterapia). Las respuestas son de tipo Likert con cuatro categorías para cada uno de los ítems, excepto para el de calidad de vida global que tiene siete. Las puntuaciones de las escalas se transforman en un rango de puntuación de 0 (mejor percepción de salud) a 100 (peor percepción de salud), excepto para la escala actividad que se interpreta al contrario. Para los ítems con ausencia de datos se siguieron las recomendaciones de los autores de la ver- sión original del RSCL ${ }^{12}$. En este estudio no se utilizó la subescala quimioterapia por no haber mostrado en estudios previos adecuadas propiedades psicométricas en enfermos no sometidos a tratamiento activo ${ }^{11}$.

Los síntomas psíquicos, ansiedad y depresión, fueron medidos a través de la versión española del Hospital Anxiety and Depresion Scale ${ }^{13}$ (HAD), que consta de 14 ítems divididos en dos subescalas, ansiedad y depresión, y cuya puntuación máxima es de 21 puntos para cada una de ellas. Según la puntuación obtenida se puede clasificar a los enfermos como normal $(<7)$, dudoso (entre 8 y 10) y potencial caso clínico (11). Esta escala ha sido ampliamente utilizada como instrumento de despistaje de ansiedad y depresión en enfermos terminales ${ }^{14}$.

Otras variables registradas fueron: edad, sexo, Karnofsky Performance Status (KPS), clase social (ingreso familiar anual bruto:I= menos de 6.000 euros; II = entre $6000 \mathrm{y}$ menos de 15.000 euros; III= entre $15.000 \mathrm{y}$ 24.000 euros; $I V=$ mas de 24.000 euros $)^{10}$, lugar de la muerte (domicilio, hospital y otros), localización del tumor primario y prescripción de fármacos (analgésicos opiáceos y otros, benzodiacepinas, hipnóticos, esteroides, antidepresivos, laxantes y antieméticos). Estas variables se tomaron del registro de las historias clínicas. Dicho registro es igual y estandarizado tanto para el EAP como para el ESAD.

Los cuestionarios RSCL y HAD fueron administrados por una profesional sanitaria previamente entrenada y ajena al estudio, en el domicilio del enfermo en tres ocasiones: a las 24 horas de entrada en el programa, a la semana y a los quince días. Los cuestionarios fueron cumplimentados por los propios enfermos o por la encuestadora con la respuesta que ellos daban.

Se consideró pérdidas a los enfermos que cambiaron de domicilio, cambiaron de nivel de atención, agravaron su estado o fallecieron. 
Para facilitar el análisis de los datos el KPS se agrupó en tres categorías (30-40; 5060 y 70-80) y la clase social en dos (I-II y IIIIV).

Para determinar el tamaño de la muestra se consideró como variable de interés principal las puntuaciones de las escalas del RSCL. Para ello se tuvo en cuenta los resultados de estudios previos ${ }^{11}$, en los que se obtuvo una desviación típica de las puntuaciones del RSCL de 20,6 y se determinó como diferencia clínicamente relevante una diferencia de puntuación de 10 puntos en al menos una escala del cuestionario entre ambos modelos de atención. El tamaño calculado fue de un mínimo de 56 enfermos por grupo para un riesgo alfa de 0,05 unilateral y beta de 0,20 .

Se utilizó análisis multivariante de la varianza (MANOVA) para evaluar las diferencias de puntuación del RSCL entre ESAD y EAP y para comparar puntuaciones del cuestionario entre visitas en cada uno de los grupos ANOVA de medidas repetidas ${ }^{15}$. También se calculó el tamaño del efecto del RSCL entre la primera visita y las restantes en cada grupo de tratamiento, por ser esta una medida estandarizada que permite detectar cambios en el estado de salud independientemente de la significación estadísti$\mathrm{ca}^{16}$. Se consideró elevado un tamaño del efecto 0,80 , moderado valores próximos a 0,50 y pequeño $<20^{17}$.

A través del test de Friedman se compararon las puntuaciones del HAD en las diferentes visitas a cada uno de los centros. Los datos fueron analizados con el paquete estadístico SPSS para Windows ${ }^{15}$, versión 6.1.2. Se consideraron valores significativos los contrastes con $\mathrm{p}<0.05$

\section{RESULTADOS}

Durante el periodo de estudio se registraron en el Área 782 POT, de los cuales 548 fueron atendidos por el ESAD y 234 por EAP. Se excluyó a 101 enfermos por ser seguidos conjuntamente entre ESAD y EAP y a 55 por presentar un KPS $<20$. Ninguno de los enfermos seleccionados mostró rechazo para participar en el estudio.

De los 217 enfermos incluidos finalmente en el estudio, 161 fueron atendidos por el ESAD y 56 por los EAP. En total aportaron enfermos al estudio 41 médicos de los 13 EAP seleccionados.

Las características personales y clínicas de los enfermos se muestran en la tabla 1. La edad media de los mismos fue de 70,6 años $(D E=10,4: \operatorname{rango}=37$ a 95) en su mayoría eran hombres y el tumor más frecuentemente padecido era el cáncer de pulmón. El 88,8\% de los enfermos presentaban un $\mathrm{KPS} \leq 60$. El $63 \%$ de sujetos tenían ingresos familiares inferiores a 15.000 euros anuales. No se encontraron diferencias estadísticamente significativas entre ESAD y EAP para estas variables (excepto tipo de tumor), ni tampoco para la presencia de metástasis a distancia que fue del 76,7\% y $76,9 \%$ respectivamente. La localización de las metástasis, que también fue similar entre ambos grupos, fue mayoritariamente en hígado, seguida de las óseas $(27 \%, 22,6 \%$ y $25 \%, 21,2 \%$ respectivamente). La supervivencia media en días fue mayor $(\mathrm{p}=0,02)$ en enfermos seguidos por EAP (media: 72,0; $\mathrm{SD}=67,6)$ en relación a ESAD (media:51,2; $\mathrm{SD}=53,6)$.

De los enfermos que iniciaron el estudio, $169(79 \%)$ cumplieron las condiciones necesarias para cumplimentar el segundo cuestionario (a la semana) y 96 (44\%) para completar el tercero (a los quince días). El motivo principal de pérdida fue la muerte $(66,7 \%)$, institucionalización o cambio de domicilio $(22,3 \%)$ y deterioro del estado funcional en el resto. El porcentaje de pérdidas y motivo de las mismas fue similar entre ESAD y EAP (57\% y 54\% respectivamente). Las pérdidas a lo largo del estudio no 
RELACIÓN DE LA CALIDAD DE VIDA CON DIFERENTES MODELOS DE ATENCIÓN DOMICILIARIA EN...

Tabla 1

Características clínicas y socio demográficas de los enfermos incluidos en estudio según tipo de centro

\begin{tabular}{|c|c|c|c|}
\hline Variable & $\operatorname{ESAD}(n-161)$ & $\operatorname{EAP}(n-56)$ & TOTAL (n-217) \\
\hline Edad: m. DE (rango) & $70.7 ; 10.7(37-95)$ & $702 ; 9.5(51-87)$ & $70.6,10.4(37-95)$ \\
\hline \multicolumn{4}{|l|}{ Sexo. n. ${ }^{\circ}$} \\
\hline Hombres & $96(59,6)$ & $31(55.3)$ & $126(58,6)$ \\
\hline Mujeres & $65(40,4)$ & $25(44.7)$ & $89(41,4)$ \\
\hline \multicolumn{4}{|l|}{ Tipo de tumor } \\
\hline Pulmón & $41(25,5)$ & $13(23,2)$ & $54(25.1)$ \\
\hline Colon, recto & $24(14,9)$ & $8(14,3)$ & $31(14,5)$ \\
\hline Estómago & $13(8,1)$ & $6(10,7)$ & $19(8.8)$ \\
\hline Otros digest. & $14(8.7)$ & $5(8.9)$ & $19(8.8)$ \\
\hline Mama & $10(6,2)$ & $7(12,5)$ & $17(7,9)$ \\
\hline Higado & $12(7.5)$ & $0(0,0)$ & $12(5,6)$ \\
\hline Próstata & $7(4.3)$ & $4(7.1)$ & $11(5,1)$ \\
\hline Otros & $33(20,5)$ & $11(19,7)$ & $43(20,0)$ \\
\hline Desconocido & $3(4,3)$ & $2(3.6)$ & $9(4.0)$ \\
\hline \multicolumn{4}{|l|}{ KPS: } \\
\hline $30-40$ & $37(23,0)$ & $13(23,2)$ & $49(22,8)$ \\
\hline $50-60$ & $103(64,0)$ & $40(71,4)$ & $142(66,0)$ \\
\hline$\geq 70$ & $21(13,0)$ & $3(5,4)$ & $24(11,2)$ \\
\hline \multicolumn{4}{|l|}{ Clase Social: } \\
\hline$<15,000$ curos & $101(62,7)$ & $39(69,6)$ & $140(65,1)$ \\
\hline$\geq 15,000$ curos & $60(37,3)$ & $16(30,4)$ & $75(34.9)$ \\
\hline
\end{tabular}

ESAD: equipo de soporte de atención domiciliaria. EAP: equipo de atención primaria; $m=$ media; $\mathrm{DE}=$ desviación estándar. KPS: Karnofsky Performance Status.

produjeron desigualdades entre grupos en cuanto a edad, sexo, estado funcional o tipo de tumor. Las características de los enfermos que no llegaron a cumplimentar el segundo cuestionario fueron similares en ambos grupos, aunque presentaron puntuaciones más altas (peor percepción de salud) en las esca-

Rev Esp Salud Pública 2003, Vol. 77, N. ${ }^{\circ} 5$ las del RSCL que los enfermos que permanecían en estudio (tabla 2 ).

Las puntuaciones del RSCL fueron inicialmente similares en ambos grupos (tabla 3). El análisis multivariante MANOVA que se realizó en dos fases (en la primera para com- 
Tabla 2

Características de los enfermos de acuerdo a su permanencia o no en el estudio

\begin{tabular}{|llc|}
\hline Variables & $\begin{array}{l}\text { Perdidas } \\
(\mathbf{n}=\mathbf{4})\end{array}$ & $\begin{array}{c}\text { Enfermos seguides } \\
(\mathbf{n}=\mathbf{1 7 0})\end{array}$ \\
\hline Edad (m, DE) & 71,$4 ; 9,1$ & 70,$3 ; 10,8$ \\
Sexo (\%) & & \\
Hombre & 63,8 & 57,6 \\
Mujer & 36,2 & 42,4 \\
KPS (\%): & & \\
$30-40$ & 31,9 & 20,6 \\
50-60 & 63,8 & 66,7 \\
Z70 & 4,3 & 12,7 \\
RSCL (m; DE) & & \\
Fisica & & $25,6,10,1$ \\
Psicologica & 30,$9 ; 11,2$ & $24,6,21,1$ \\
Actividad & 29,$1 ; 216$ & 34,$8 ; 25,7$ \\
Global & 25,$4 ; 21,5$ & $49,4,24,3$ \\
Fatiga & 59,$9 ; 27,9$ & 38,$1 ; 17,8$ \\
Dolor & 48,$7 ; 15,7$ & 22,$1 ; 18,1$ \\
Sintomas gástricos & 24,$9 ; 18,9$ & 23,$5 ; 17,2$ \\
\hline
\end{tabular}

m = media; DE= desviación estándar. KPS: Karnofsky Performance Status. RSCL: Rotterdam Symptom Check List

parar las puntuaciones del RSLC a la semana y en la segunda para compararlas a los quince días) mostró alta significación estadística ( $\mathrm{p}=0,0001$ para la prueba Wilk's Lambda). Las puntuaciones del RSCL mostraron mejoría (menor puntuación) en los enfermos atendidos por el ESAD, en relación a los atendidos por EAP, en la segunda visita en todas las dimensiones, excepto en la psicológica y en la relativa a actividad, aunque solo fueron significativas las diferencias en las escalas física, global y dolor $(\mathrm{p}<0,05)$, como se aprecia en la tabla 3 . Aunque las puntuaciones fueron, en general, también mejores en la tercera visita para los enfermos seguidos por ESAD, excepto para la escala física, no se encontraron diferencias estadísticamente significativas. Las covariables introducidas: edad, sexo, KPS, tiempo de supervivencia y tipo de tumor (codificado en: pulmón, colon, mama y otros) no produjeron cambios estadísticamente significativos, por lo que no se incluyeron en el modelo final.

El análisis de cada grupo mostró, en los enfermos seguidos por ESAD, una mejora significativa en la puntuación de las escalas física, global y dolor en la segunda visita y sólo en la escala dolor en la tercera visita. En los enfermos seguidos por EAP esta mejoría sólo se observó al final del estudio en las escalas dolor y gastrointestinal. El tamaño del efecto fue moderado (mayor de 0,30) para todas las escalas (excepto psicológica y actividad, para las que fue menor) en la segunda visita, y para la física y dolor en la última visita en ESAD. En el caso de EAP el tamaño del efecto fue moderado para las escalas física, gastrointestinal y fatiga en la

Rev Esp Salud Pública 2003, Vol. 77, N. ${ }^{\circ} 5$ 
RELACIÓN DE LA CALIDAD DE VIDA CON DIFERENTES MODELOS DE ATENCIÓN DOMICILIARIA EN...

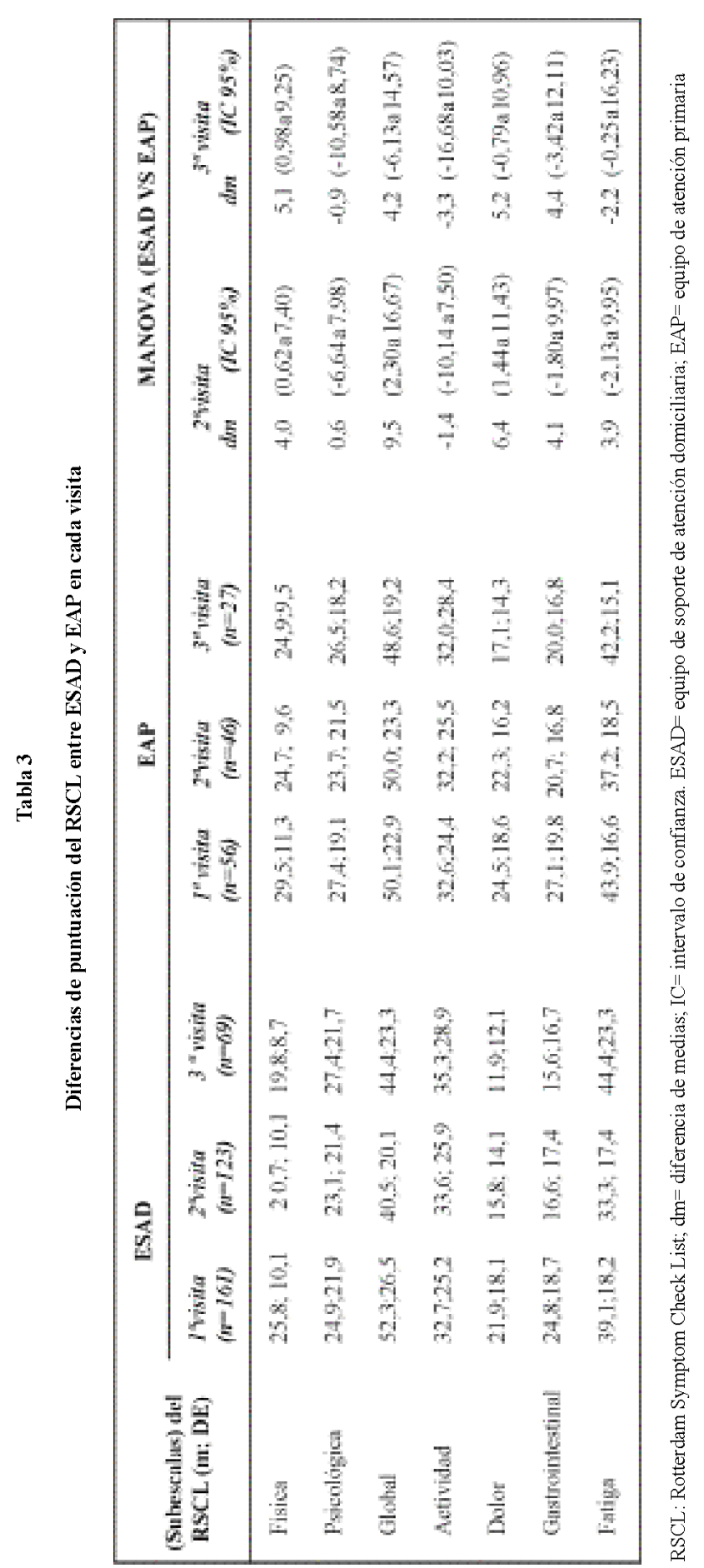

Rev Esp Salud Pública 2003, Vol. 77, N. ${ }^{\circ} 5$ 
Yolanda Agra Varela et al.

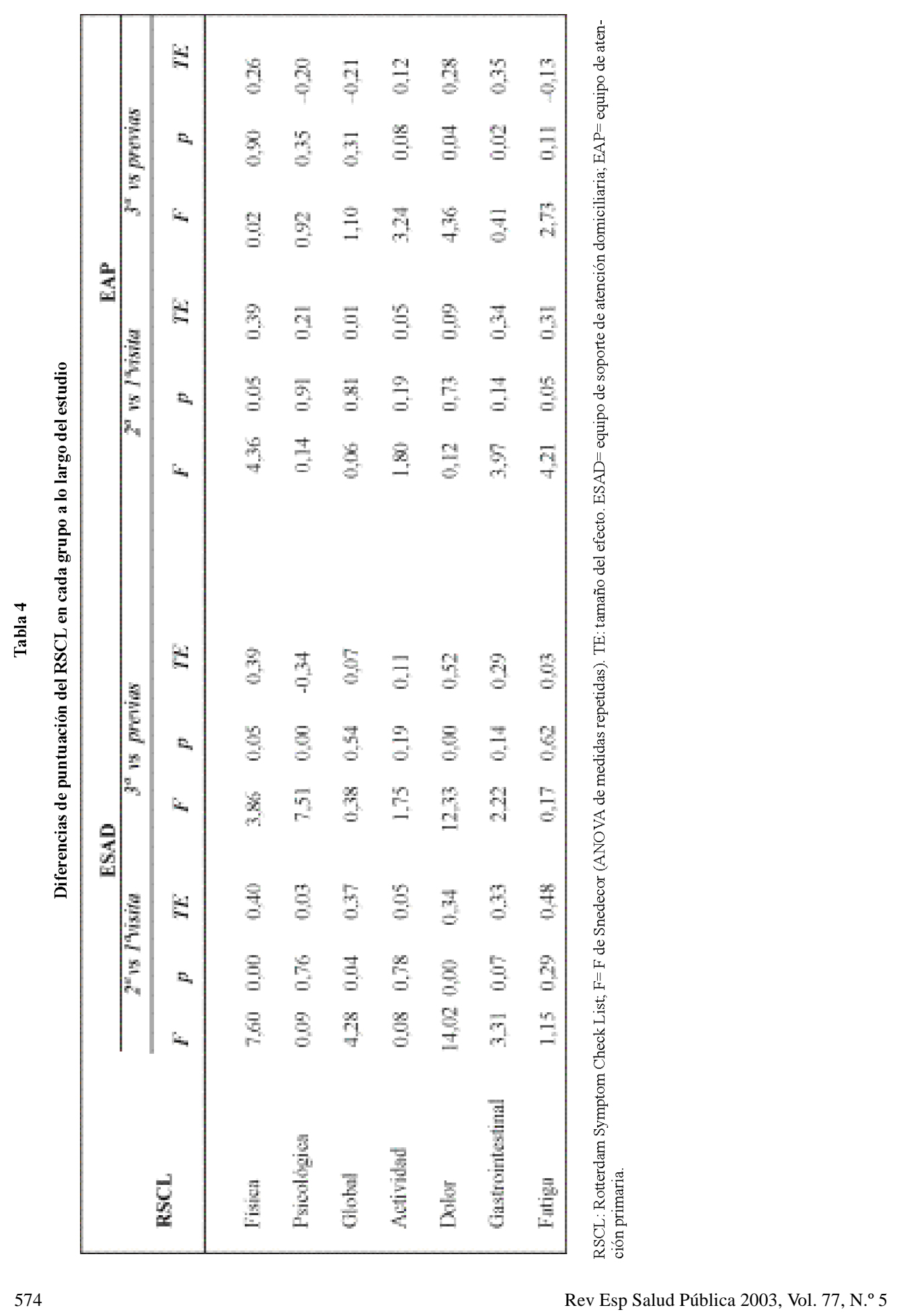


Tabla 5

Puntuaciones del HAD según centro en cada visita

\begin{tabular}{|c|c|c|c|c|c|c|c|c|}
\hline \multirow[b]{2}{*}{ Escalas del HAD } & \multicolumn{3}{|c|}{ ESAD } & \multicolumn{5}{|c|}{ EAP } \\
\hline & $\begin{array}{l}\left.l^{*} \text { visita }\right) \\
(n=16 I)\end{array}$ & $\begin{array}{l}2^{a} \text { visita } \\
(n=123)\end{array}$ & $\begin{array}{c}3^{*} \text { visita } \\
\quad(n=69)\end{array}$ & $p$ & $\begin{array}{l}I^{*} \text { visifa } \\
(n=50)\end{array}$ & $\begin{array}{l}2^{*} \text { visita } \\
(u=\$ 5)\end{array}$ & $\begin{array}{c}3 * \text { visita } \\
(n=27)\end{array}$ & $p$ \\
\hline \multicolumn{9}{|l|}{ Ansicdad (\%) } \\
\hline 0.7 & 76.4 & 78.9 & 85,5 & 0,92 & 83,3 & 80,0 & 75,0 & 0.25 \\
\hline $8-10$ & 13,7 & 13,8 & 10,1 & & 11,1 & 8,9 & 25,0 & \\
\hline 211 & 9.9 & 7,3 & 4,3 & & 5,6 & 11.1 & . & \\
\hline \multicolumn{9}{|l|}{ Depresión (\%) } \\
\hline 0.7 & 50,0 & 55,6 & 60,3 & 0,08 & 46,2 & 46,3 & 40,0 & 0,11 \\
\hline $8-10$ & 18,6 & 13,7 & 12,7 & & 23,1 & 17,1 & 24.0 & \\
\hline$\geq 11$ & 31,4 & 30,8 & 270 & & 30,8 & 36.6 & 360 & \\
\hline
\end{tabular}

ESAD: equipo de soporte de atención domiciliaria. EAP: equipo de atención primaria. HAD: Escala Ansiedad Depresión. P= significación del test de Friedman.

segunda visita y solo gastrointestinal en la última visita (tabla 4).

El porcentaje total de enfermos con ansiedad o depresión, según la escala HAD, fue del $11 \%$ en ESAD y del $32 \%$ en EAP respectivamente. Aunque no se encontraron diferencias estadísticamente significativas, a lo largo del estudio el porcentaje de enfermos clasificados con ansiedad o depresión disminuyó en frecuencia en ESAD y aumentó en EAP (tabla 5).

La muerte en el domicilio fue del 58,8\%, para el total de enfermos y ligeramente diferente entre grupos $(56,6 \%$ en ESAD y $65,8 \%$ en EAP). La prescripción de fármacos analizados presentó diferencias entre grupos para analgésicos no opiáceos (92\% en ESAD y $76 \%$ en EAP), opiáceos (78,3\% y $87 \%)$, benzodiacepinas $(51,6 \%$ y $37 \%)$ y esteroides $(62,1 \%$ y $48,1 \%$ respectivamente).

\section{DISCUSIÓN}

La medición de la calidad de vida en la evaluación de programas de intervención sanitaria permite conocer desde la perspecti- va del enfermo, las ganancias o pérdidas en salud obtenidas con los servicios prestados y ayuda a la toma de decisiones en la planificación sanitaria.

Los datos de este estudio muestran una mejor percepción de salud en los enfermos seguidos por el ESAD en relación a los enfermos seguidos por EAP, especialmente en la primera semana de inclusión en el programa. Esta mejoría se ha manifestado para las escalas física, global y dolor del RSCL.

La interpretación clínica de estos resultados está relacionada con el cambio clínico mínimamente importante a considerar. En el caso de cuestionarios de calidad de vida relacionada con la salud este cambio se basa, entre otros parámetros, en el tamaño del efecto y en el cambio de puntuación del cuestionario en relación a una pregunta general de salud ${ }^{16}$. El tamaño del efecto en este estudio ha sido moderado en la segunda visita para todas las escalas del RSCL en el grupo ESAD, excepto en la psicológica y en la relativa a la actividad, y en tres de ellas en el grupo EAP. El tamaño del efecto se ve influenciado, no obstante, por la variación en el número de sujetos antes y después de la 
intervención, por lo que no ha tenido la magnitud que podría haberse esperado. Por otro lado, en estudios de seguimiento ${ }^{19}$ se considera que un cambio medio aproximado de 0,5 en la puntuación de una pregunta general de salud, como la que compone la escala global del RSCL, se corresponde con una diferencia clínicamente importante. La escala global ha presentado en el grupo ESAD una diferencia de puntuación (mejor percepción de salud) de 11,8 en la primera semana y de 7,9 en la segunda, lo cual apoya la significación clínica de los resultados.

A lo largo del estudio Las características clínicas y sociodemográficas de los enfermos (edad, sexo, tipo de tumor, KPS y clase social) no presentaron variaciones estadísticamente significativas entre ambos grupos, por lo que no parece que puedan haber influido en las diferencias encontradas. Sí se observaron diferencias entre grupos en el tiempo medio de supervivencia, debidas en parte a que el ESAD, como unidad especializada, atiende a enfermos con peor pronóstico y a que tres enfermos del grupo EAP presentaron valores extremos con tiempos medios de supervivencia mayores de 250 días. No obstante, la inclusión del tiempo de supervivencia en el modelo MANOVA no introdujo modificaciones relevantes en las diferencias encontradas entre grupos.

Los resultados aquí mostrados están en consonancia con otras publicaciones que refieren mejoría de la calidad de vida en enfermos seguidos en unidades específicas de cuidados paliativos ${ }^{20}$. Sin embargo cuando se comparan varios modelos asistenciales los resultados de la mayoría de los estudios muestran que no existen diferencias relevantes entre cuidados especializados y estandarizados $^{21,22}$, y si existen es a favor de una mayor satisfacción global y mejor control del dolor por parte de equipos especializados en cuidados paliativos ${ }^{4,23}$ como también muestra el presente trabajo.

El que las diferencias detectadas en la primera semana del estudio no se mantengan a lo largo del mismo podría deberse, en parte, al insuficiente numero de sujetos, lo que impide detectar diferencias entre grupos. En este sentido, si bien el tamaño de la muestra se mantiene en el límite necesario en el grupo ESAD, a lo largo del estudio, el menor reclutamiento de sujetos en el grupo EAP y el número de enfermos perdidos producen una reducción del poder estadístico para detectar diferencias. En este estudio el $44 \%$ de los sujetos sigue en programa a los 15 días de seguimiento, siendo el $90 \%$ de las perdidas debidas a éxitus o deterioro del estado funcional. La medición de los resultados en cuidados paliativos de enfermos oncológicos es difícil de realizar por la perdida importante de sujetos, sobre todo en las primeras semanas de seguimiento, como ya ha sido comentado en otros trabajos ${ }^{24,25}$.

El número de enfermos en el grupo EAP fue inferior al de ESAD debido por un lado al menor número de enfermos atendidos durante el periodo de estudio y a la baja participación de los médicos seleccionados. A pesar de haber mantenido dos reuniones y contactos personales con ellos, probablemente no se sintieron suficientemente motivados ya que no se les ofreció ningún tipo de compensación por participar en el estudio.

Los cuestionarios aquí utilizados cumplen los requerimientos psicométricos necesarios en la investigación de enfermos con cán$\operatorname{cer}^{16}$. La versión española del RSCL ha mostrado validez, fiabilidad y sensibilidad a los cambios en un estudio previo realizado con POT de características similares a los incluidos en este estudio ${ }^{11}$. Además el RSCL se comporta como una escala de síntomas, breve y fácil de cumplimentar, por lo que resulta de gran utilidad en enfermos con cáncer sometidos a tratamiento. El HAD es una escala que ha mostrado utilidad en cuidados paliativos por ser aceptable para los enfermos y permitir detectar alteraciones psicológicas susceptibles de tratamiento ${ }^{14}$.

La frecuencia de ansiedad y depresión referida por los enfermos estudiados se 
corresponden con la ofrecida en otras series de enfermos con cáncer avanzado ${ }^{14,27}$. Aunque se observó una disminución en ESAD, en la frecuencia de enfermos con estos síntomas durante el seguimiento, estas diferencias no fueron estadísticamente significativas. En ambos grupos la escala psicológica del RSCL presentó estabilidad o empeoramiento de sus puntuaciones lo que parece apoyar la idea, ya señalada en otros trabajos, que en este tipo de enfermos los síntomas psíquicos empeoran con el avance de la enfermedad y presencia de síntomas físi$\cos ^{11,27}$.

La prescripción de fármacos, que fue adecuada a las recomendaciones actuales ${ }^{28}$, presentó diferencias en cuanto a la utilización de analgésicos, benzodiacepinas y corticoides, fármacos de uso prioritario para paliar los síntomas más frecuentes de estos enfermos $^{28}$. Aunque algún estudio muestra una mayor prescripción de opiáceos por equipos especializados que en centros de atención primaria ${ }^{2}$ esto no se ha confirmado en el presente trabajo. La adecuación de dosis, pauta, asociación de analgésico y utilización de coanalgésicos (fármacos sin indicación analgésica pero utilizados como tales en situaciones especificas, como los corticoides) no ha sido analizada en este estudio y podría ser un factor que explicase el mejor control del dolor en enfermos seguidos por el ESAD. Hay que recordar que los médicos de atención primaria atienden anualmente entre uno y tres enfermos oncológicos terminales al $\mathrm{año}^{7}$, lo cual no garantiza necesariamente un adecuado manejo de los mismos.

Cuando se le pregunta a los enfermos con cáncer dónde preferirían morir, la respuesta más frecuente es que en su propio domici$\operatorname{lio}^{29}$ y por ello esta variable se ha relacionado con la calidad de vida, motivo por el cual ha sido registrada en este trabajo. El porcentaje de enfermos que murieron en su domicilio fue mayor al referido por países de larga tradición de medicina paliativa ${ }^{29}$. Por razo- nes socioeconómicas y culturales, parece que la muerte en casa se produce con mas frecuencia en nuestro país que en otros del entorno. El hecho de que la mujer, principal cuidadora de estos enfermos, no llegue a los niveles de incorporación laboral de los países del resto de la Comunidad Económica Europea $^{30}$, podría justificar en parte este resultado. La mayor dificultad de los médicos de familia para derivar a los enfermos a centros de cuidados mínimos podría explicar también el mayor número de muertes en casa en el grupo EAP.

Las recomendaciones para aconsejar una medida sanitaria de intervención deben basarse en evidencias halladas a través de ensayos clínicos ${ }^{5}$, sin embargo esto no ha sido posible por razones de planificación y organización sanitaria en nuestro medio. No obstante parece poco probable que existiera «contaminación psicológica» debido a que los médicos participantes desconocían los objetivos del estudio y los enfermos en su domicilio ignoraban el cuidado recibido por los otros enfermos.

Los médicos de atención primaria deben ser responsables del cuidado paliativo de los enfermos en el contexto de equipos multidisciplinares, por lo que parece necesario ofrecerles formación específica y apoyo en el manejo de los enfermos en situación terminal $^{31,32}$. Aunque en este estudio se excluyó a los enfermos que habían sido tratados en conjunto por médicos de atención primaria y profesionales del ESAD, la mayor parte de médicos del Área donde se realizó este estudio habían tenido acceso a formación previa en cuidados paliativos, lo que podría explicar en parte que no se hayan encontrado mayores diferencias entre ambos grupos. De confirmarse estos datos en futuros estudios quizás podría plantearse que no se trate de determinar un servicio exclusivo de cuidados paliativos sino mas bien de diseñar planes de actuación conjunta entre todos los posibles proveedores para, manteniendo el principio de equidad, mejorar la 
calidad de vida de los enfermos terminales $^{33}$.

En resumen, los datos de este estudio muestran que los enfermos oncológicos terminales del Área 4 de Madrid seguidos por el ESAD presentan mejor percepción del estado de salud, reflejado en una mejora de las escalas física, global y dolor del RSCL en relación a los enfermos seguido por los EAP a la semana de su inclusión en programa. Sin embargo las diferencias encontradas no han sido tan grandes como se esperaba, ni se han mantenido a lo largo del estudio probablemente debido al gran número de pérdidas sufridas y a la formación previa en cuidados paliativos de los médicos de EAP. Esto hace que debamos tomar los resultados con precaución, aunque debido a que no hemos encontrado ningún estudio similar en atención primaria en nuestro medio, creemos suponen una aportación novedosa sobre la evaluación de la asistencia sanitaria a domicilio a enfermos oncológicos terminales. Serían recomendables estudios de base poblacional más amplia para conocer de forma más precisa la efectividad y eficiencia de los programas de atención domiciliaria a enfermos oncológicos terminales actualmente en marcha en atención primaria en España. En estos estudios se podría también determinar la calidad de cuidados recibidos por enfermos atendidos conjuntamente por diferentes proveedores de salud.

\section{BIBLIOGRAFÍA}

1. Doyle D, Hanks G, Mac Donald N. Oxford Textbook of Palliative Medicine. 2nd Edition. New York Oxford University Press; 1997.

2. Lamí U, Kosunen E, Kellokumpu-Lehtinen P. Palliative cancer care in two health centres and one hospice in Finland. Support Care Cancer 2000; 9:25-31.

3. Smeenk FWJM, van Haastregt JMC, de Witte LP, Crebolder HFJM. Effectiviness of home care programmes for patients with incurable cancer on their quality of life and time spent in hospital: systematic review. BMJ 1998;316: 1939-44.
4. Fordham S, Dowrick. Is care of the dying improving? The contribution of specialist and non-specialist to palliative care. Fam Pract 1999; 16:573-579.

5. Higginson IJ. Evidence based palliative care. BMJ 1999;319:462-3.

6. Smeenk F, van Haastregt JCM, deWitteL P, Crebolder FJM. Effectiveness of home care programmes for patients with incurable cancer on their quality of life and time spent in hospital: systematic review. BMJ 1998; 316:1939-44.

7. Insalud Madrid Área 4. Programa de Cuidados Paliativos a Domicilio. Madrid: Dirección de Atención Primaria Área 4. $2^{a}$ ed: Insalud;1997.

8. Karnofsky DA, Abelmann WH, Craver LF et al. The use of nitrogen mustards in the palliative treatment of carcinoma. Cancer 1948; 1:623-56.

9. Pfeiffer E. A short portable mental status questionnaire for the assessment of organic brain deficit in elderly patients. J Am Geriatrics Soc 1975; 23:433441

10. Agra Y, Badià X. Spanish version of the Rotterdam Symptom Check List: cross-cultural adaptation and preliminary validity in a sample of terminal cancer patients. Psycho-Oncology 1998;7:229-39.

11. Agra Y, Badià X. Evaluación de las propiedades psicométricas de la versión española del Rotterdam Symptom Check List para medir calidad de vida en enfermos oncológicos. Rev Esp Salud Pública. 1999, 73: 35-44.

12. de Haes JC, Olschewski M, Fayers P, Visser MRM, Cull A, Hopwood P et al. Measuring the quality of life of cancer patients with the Rotterdam Symptom Check List (RSCL): A manual. Northen Centre for Health Care Research $(\mathrm{NCH})$. The Netherlands: University of Groningen; 1996.

13. Tejero A, Guimerá EM, Farré JM. Uso clínico del HAD (Hospital Anxiety and Depression Scale) en población psiquiátrica: Un estudio de su sensibilidad, fiabilidad y validez. Rev Psiquiatr Fac Med Barc 1986; 13:233-8.

14. Holtom N, Barraclough J. Is the Hospital Anxiety and Depression Scale (HADS) useful in assessing depression in palliative care? Palliat Med 2000; $14: 219-20$

15. Staquet MJ, Hays RD, Fayers PM. Quality of life assessment in clinical trials. Methods and Practice. Oxford University Press. Oxford New York; 1998.

16. Hays RD, Anderson R, Revicki DA. Psychometric evaluation and interpretation of health-related qua-

Rev Esp Salud Pública 2003, Vol. 77, N. ${ }^{\circ} 5$ 
lity of life data. In: shumaker SA, Berzor RA. The International Assessment of Health Related Quality of Life: Theory, Translation Measurement and Analysis. Oxford: Rapid Communications of Oxford Ltd; 1995.p.103-14

17. Kazis L, Anderson J, Meenan R. Effect Sizes for interpreting changes in health status. Medical Care 1989; 27 Suppl: 178-89.

18. SPSS [programa informático]. Versión 6.1.2. para Windows en español: Microsfot Corporation;1995.

19. Badià, Carné X. La evaluación de la calidad de vida en el contexto del ensayo clínico. Med Clin 1998;110:550-6.

20. Cohen R, Boston P, Mount BM, Porterfield P. Changes in quality of life following admission to palliative care units. Pall Med 2001; 15:363-71.

21. Eran J, Higginson IJ. Do specialist palliative care teams improve outcomes for cancer patients? A systematic literature review of the evidence. Pall Med 1998;12:317-32.

22. Jordhoy MS, Fayers P, Loge JH, Ahlner-Elmqvist $\mathrm{M}$, Kaasa S. Quality of life in palliative cancer care: results from a cluster randomized trial. J Clin Oncol 2001; 19: 3884-94

23. Salisbury C, Bosanquet N, Wilkinson EK, Franks PJ, Kite S, Lorentzon M et al. The impact of different models of specialist palliative care on patients'quality of life: a systematic review. Pall Med 1999; 13: 3-17.

24. McWhinney IR, Bass MJ, Donner A. Evaluation of a palliative care service: problems and pitfalls. BMJ 1994:309:1340-42.
25. Donnelly S, Rybicki L, Walsch D. Quality of life measurement in the palliative management of advanced cancer. Supp Care Cancer 2001; 9:361-5.

26. Weiss S, Emanuel L, Fairclough D, Emanuel E. Understanding the experience of pain in terminally ill patients. The Lancet 2001; 357:1311-5.

27. Plumb B, Holland J. Comparative studies of psychological function in patients with advanced cancer I: Self-reported depressive symptoms. Psychosom Med 1997;39:264-76.

28. Whitecar P, Jonas P, Clasen M. Managing Pain in the Dying Patient. Am Fam Phys 2000;61:755-64.

29. Higginson I, Astin P, Dolan S. Where do cancer patients die? Ten-year trends in the place of death of cancer patients in England. PallMed 1998; 12:353-63.

30. Seasonally adjusted unemployment rate. Disponible en: http://europa.eu.imt/comm/eurostat/

31. Brow JB, Sangster M, Swift J. Factors influencing palliative care. Qualitative study of family physicians'practices. Can Fam Physician 1998; $44: 1028-4$.

32. Shipman C, Addington-Hall J, Barclay S, Briggs J, Cox I, Daniels L, Millar D. Educational opportunities in palliative care: what do general practitioners want? Pall Med 2001; 15: 191-6.

33. Hanks GW, Robbins M, Sharp D, Forbes K, Done K, Peters TJ et al. The imPaCT study: a randomised controlled trial to evaluate a hospital palliative care team. Br J Cancer 2002;87: 733-9. 